\title{
High Frame Rate Contrast Enhanced Ultrasound Imaging of Lymphatic Vessel Phantom
}

\author{
Jiaqi Zhu, Shengtao Lin, Sevan Harput, Matthieu Toulemonde, Chee Hau Leow, Meng-Xing Tang \\ Department of Bioengineering, Imperial College London, London, United Kingdom \\ Mengxing.tang@imperial.ac.uk
}

\begin{abstract}
Contrast enhanced ultrasound (CEUS) shows great potentials for visualising lymphatic vessels and identifying sentinel lymph nodes (SLN) which aid the diagnosis of breast cancer. However, current CEUS imaging techniques have some limitations: 1) Nonlinear tissue artefacts result in false bubble signals and reduce the image contrast; 2) Low spatial and temporal resolution limits the amount of information that can be captured by CEUS; 3) The slow lymph flow makes Doppler based approaches less effective. These limitations have prevented the accurate visualisation of lymphatic vessels and SLN. This work demonstrates the potential of high frame-rate (HFR) CEUS in detecting lymphatic vessels, and investigates the effects of flow velocity and ultrasound pressure on HFR CEUS imaging of lymph vessels in terms of image contrast and bubble signal persistence over time. It is shown that under slow flow, ultrasound amplitude has significant impacts on both image contrast and signal persistence.
\end{abstract}

Keywords-Lymphatic vessel imaging; High frame rate ultrasound ; Microbubble disruption; Contrast agents; Singular value decomposition

\section{INTRODUCTION}

Sentinel lymph node (SLN) is the first axillary lymph node that receives the lymphatic fluid from breast tumour. Hence, the presence of metastases in SLN is the most important indicator for breast cancer staging [1]. Preoperative, non-invasive detection and characterisation of SLN in breast cancer patients are valuable for the diagnosis and management of the disease.

Recently, the potential of using contrast enhanced ultrasound (CEUS) to preoperatively identify lymphatic vessels and SLN in breast cancer patients has been demonstrated. CEUS is a new imaging technique which utilises microbubble as contrast agents to allow better visualisation of vasculature and flow. When microbubbles are exposed to ultrasound field, they produce backscattered signals containing fundamental and harmonic components, which greatly enhance ultrasound echoes. Microbubbles have been widely used in clinical diagnosis and have reported few side effects or complications. The ability of preoperative investigation of SLN makes CEUS a promising axillary nodal staging method. There are several studies on the use of CEUS for the lymphatic system. The technique was first described by Goldberg et al. [2] in several swine melanoma model. Sever et al. [3] applied CEUS for the visualisation of lymphatic vessels and SLNs in patients with diagnostic breast cancer. In their most recent study [4], CEUS was used to guide percutaneously SLN biopsy after preoperative detection of SLN in 295 breast cancer patients, among which a secondary surgical procedure was avoided in 29 patients.

However, nonlinear tissue artefacts are significant in lymphatic imaging. In CEUS, nonlinear echoes arising from microbubble oscillations can be used to distinguish microbubble from tissue signals using different imaging techniques such as pulse inversion (PI) and amplitude modulation (AM). However, nonlinear echoes can also be produced by nonlinear propagation of ultrasound in tissue or bubble cloud [5]. The nonlinear tissue artefact misrepresents tissue as microbubbles, leading to reduced image contrast and inaccuracies in bubble tracking and SLN identification. It also increases variability which makes it susceptible to greater operator dependency. The slow flow in the lymphatic system (typically around $4 \mathrm{~mm} / \mathrm{s}$ in axillary lymphatic vessels) makes clutter filter difficult in Doppler based approaches.

Two recent advances in ultrasound, HFR ultrasound imaging and spatiotemporal clutter filtering, can help to overcome the above problem. Compared to conventional line-by-line focus imaging, HFR imaging with frame rate typically higher than 1000 frames per second can be achieved based on unfocused plane wave imaging. Furthermore, coherent summation of varied angle plane wave transmission is able to improve the image quality and make up for the lack of focus transmission [6]. Enhanced contrast is achieved by plane wave images at the same disruption ratio of focus images, when detecting microbubbles [7]. Thanks to the dramatic increase in the number of temporal samples in ultrafast plane wave imaging, advanced spatiotemporal analysis (STA) such as singular value decomposition (SVD) filtering can be used to remove dominant artefact in blood flow imaging arising from slow moving tissue [8]. Unlike traditional temporal high pass filter, STA makes use of the distinct spatiotemporal coherence of blood $/ \mathrm{microbubbles}$ and tissue signals. The effectiveness of STA for differentiating between small vessels with slow flow and significant tissue motion has been demonstrated in ultrafast Doppler blood imaging [9] and HFR CEUS imaging [10].

In this study, we demonstrate the application of ultrafast CEUS and spatiotemporal filtering for visualisation of slow flow on a lymphatic mimicking phantom. In-vitro experiments for simulating lymphatic vessel imaging are designed to understand the impacts of lymph flow velocities and ultrasound pressures on HFR CEUS. Our study shows the need of optimising ultrasound pressure for different flow velocities in order to obtain optimal image quality. 


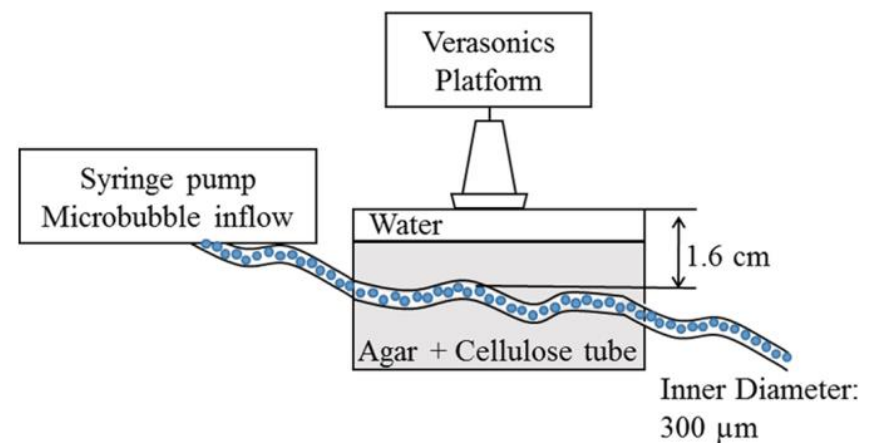

Fig.1. In-vitro set-up for investigating the effects of flow velocity and ultrasound pressure. Tissue-mimicking phantom with a $300 \mu \mathrm{m}$ tube was designed to simulate the size and depth of lymphatic vessel. Microbubbles were injected by a syringe pump to achieve velocities in range of lymph flow.

\section{METHODS}

\section{A. Phantom set-up and ultrasound acquisition}

A lymph vessel mimicking phantom (Fig. 1) was made consisting of a $300 \mu \mathrm{m}$ tube inside a tissue-mimicking phantom. The phantom was made of agar containing glass beads as scatterers. The diameter of the tube was chosen according to the average size of afferent lymphatic vessels [11]. The depth of the tube was set at $1.6 \mathrm{~cm}$ from the top surface, which mimics the average depth between the axillary lymphatic vessel and the skin [12]. Microbubbles of concentration $\sim 2.5 \times 10^{7} / \mathrm{mL}$ were injected using a syringe pump. The microbubbles used for all the experiments were homemade lipid-shell microbubbles filled with decafluorobutane (DFB) which were manufactured according to the formula provided by S. Lin [13].

HFR plane wave imaging were acquired using a research platform (Verasonics Vantage), a linear probe L12-3v (Verasonics, USA; pitch $0.2 \mathrm{~mm}$ ), and a 5-angle compounding PI plane wave imaging sequences. The transmitted pulses were centred at $4 \mathrm{MHz}$ and the frame rate was $1000 \mathrm{~Hz}$ for all the ultrasound (after compounding) acquisitions. The acquired radio frequency (RF) data were beamformed to give 1000 frames of compounded PI images per second which were then processed by STA based on SVD. Components corresponding to higher singular values, which represent tissue signals, were filtered out. The SVD cut-off thresholds were chosen empirically so as to obtain the optimal CTR.

\section{B. Ultrasound experiment: effect of flow rate and pressure}

The effect of different flow velocities in lymphatic vessels and the different degrees of microbubble disruption were investigated. An adjustable syringe pump connected to one side of the tube was used to inject and control the flow velocities of microbubbles at 4 and $10 \mathrm{~mm} / \mathrm{s}$ to simulate lymph flow. These flow velocities were chosen according to the different lymph flow rate found in the main lymphatic trunks of human [14]. Axillary lymphatic vessels are distributed in the subclavian lymph trunk with an average flow rate of $0.7 \mathrm{~mm}^{3} / \mathrm{sec}$ (converted to flow velocity $4 \mathrm{~mm} / \mathrm{s})$. Two ultrasound pressures $(\mathrm{MI}=0.03$, 0.22 ) were applied to obtain different levels of microbubble disruption. The lower MI value (0.03) is currently used in contrast imaging to avoid microbubble destruction. The higher one $(0.22)$ was applied to introduce microbubble disruption.
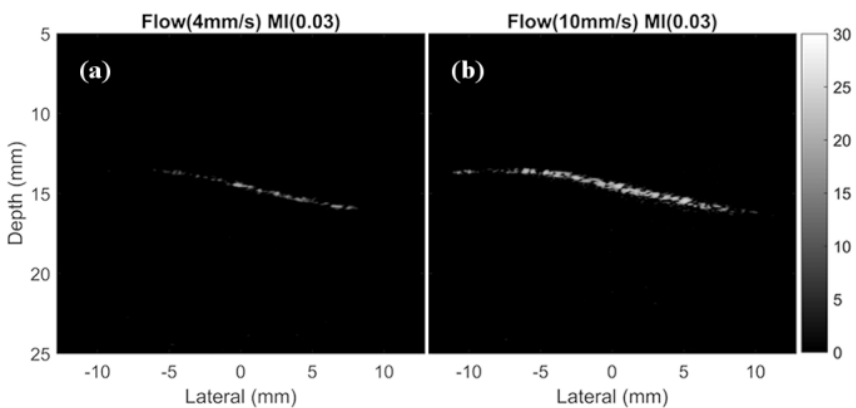

Fig.2. Qualitative evaluation of impacts of flow velocity. Images after STA on PI at (a) flow velocity $=4 \mathrm{~mm} / \mathrm{s}$ (b) flow velocity $=10 \mathrm{~mm} / \mathrm{s}$. Both images are with $\mathrm{MI}=0.03$.

\section{Optical experiment}

Microbubble behaviour under different ultrasound amplitudes was further investigated in optical experiments. High speed optical camera (FASTCAM SA5, Photron, USA), which recorded optical images at $12 \mathrm{k}$ frames per second, was synchronised with ultrasound image sequences for observing microbubble behaviour. The optical path was perpendicular to the ultrasound path to reduce the effect from objective on ultrasound wave. Unlike the phantom used in ultrasound experiments, a $200 \mu \mathrm{m}$ optically and acoustically transparent cellulose-tube filled with microbubble solution of the same concentration $\left(\sim 2.5 \times 10^{7} / \mathrm{mL}\right)$ was placed at the focal depth of microscope.

\section{RESULTS AND DISCUSSION}

\section{A. Ultrasound experiment}

The effect of flow velocities and ultrasound pressures on the lymphatic vessel imaging is evaluated both qualitatively and quantitatively.

Qualitative evaluation is performed by visual observation of the images obtained from the STA of the PI images. Fig. 2 compares the images of two different flow velocities $(4 \mathrm{~mm} / \mathrm{s}$ and $10 \mathrm{~mm} / \mathrm{s}$ ) at a fixed MI of 0.03 . Improved contrast signals can be clearly observed in the case of the higher flow velocity. Fig. 3 compares images obtained using two different MIs $(0.03$ and 0.22 ) at a fixed flow velocity of $4 \mathrm{~mm} / \mathrm{s}$. At $50 \mathrm{~ms}$ during the acquisition, better contrast signals are observed at higher MI (Fig. 3b) rather than low MI (Fig. 3a). Furthermore, towards the later stage of the acquisition at $550 \mathrm{~ms}$, we observe comparable bubble signals between low MI (Fig. 3c) and higher MI (Fig. $3 d)$.

Quantitative evaluation is conducted using two measures. The first is contrast-to-tissue ratio (CTR) which takes the ratio of the mean intensities of the vessel region over that of the surrounding tissue region. The mean CTR is calculated by averaging the individual CTR of each frame across a time interval of $1 \mathrm{~s}$. Fig. 4 shows the effect of different flow velocities and MIs on the mean CTR. We observe that an increase in the flow velocities results in a corresponding increase in mean CTR for both MI values, which is consistent with the qualitative results shown in Fig. 2. This is because the distinction between microbubble and tissue with STA relies on the different spatiotemporal coherence levels. The temporal characteristics and spatial distribution of fast flowing microbubbles and static 

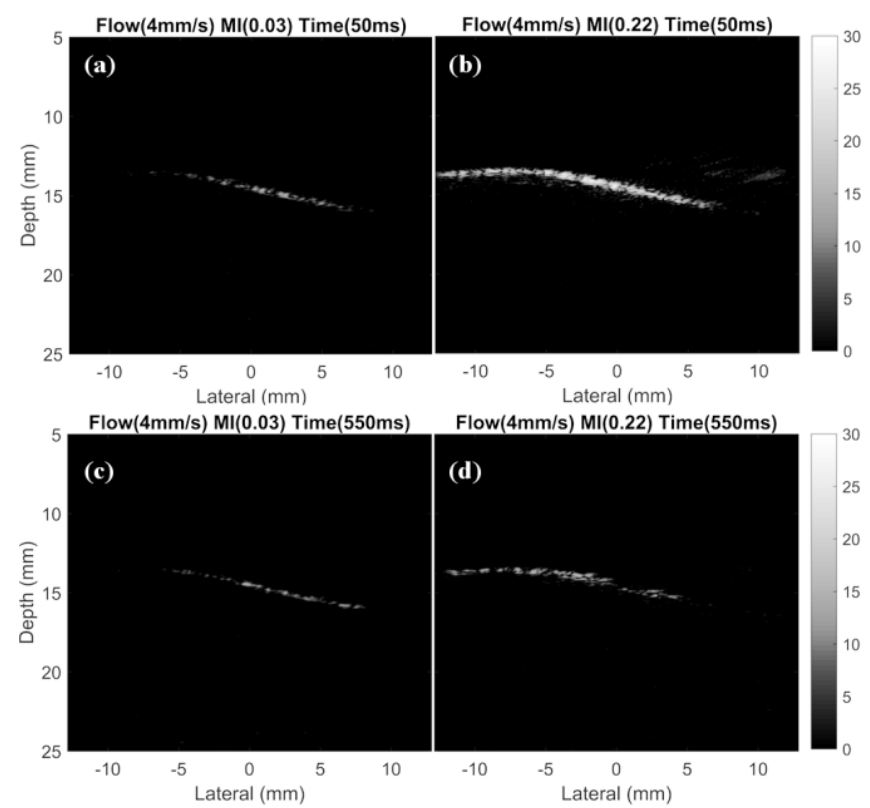

Fig.3. Qualitative evaluation of impacts of ultrasound pressure. Images after applying STA on PI at flow velocity $=4 \mathrm{~mm} / \mathrm{s}$ with (a) $\mathrm{MI}=0.03$, time $=50 \mathrm{~ms}$; (b) $\mathrm{MI}=0.22$, time $=50 \mathrm{~ms}$; (c) $\mathrm{MI}=0.03$, time $=550 \mathrm{~ms}$; (d) $\mathrm{MI}=0.22$, time $=550$ ms.

tissue speckles differ widely, resulting in enhanced CTR. But for the slow flowing microbubbles, the difference from static tissue is not significant, resulting in low CTR.

Fig. 4 also shows that higher MI increases mean CTR at both flow velocities. This result agrees with the visual observation in Fig. 3a and $3 \mathrm{~b}$. This is because increase in ultrasound pressure introduces varied microbubble disruption behaviour, especially with large amount of microbubbles at the beginning of the acquisition. These microbubble changes are able to produce dissimilarity in temporal characteristics and spatial distribution (i.e. spatiotemporal coherence) between microbubble and tissue signals, resulting in improved efficiency of STA. However, mean CTR averages across a large time interval and does not show the contrast quality over time. For instance, it does not reflect the comparable result shown in Fig. 3c and $3 \mathrm{~d}$ which occurs at the later stage of the acquisition.

To this end, we introduce a second measure, integral of normalised intensity (INI), which quantifies the ability to retain contrast signals over time. To compute INI for a video sequence, we first obtain the intensities of the vessel region over the acquisition time of $1 \mathrm{~s}$. The intensities are then normalised by the vessel intensity of the first frame of the video so that the intensity at $\mathrm{t}=0$ is 1 for all video sequences. INI is given by the integration of normalised intensities over the acquisition time of $1 \mathrm{~s}$. INI is a measure that indicates contrast signal persistence over time. Fig. 5 shows the effect of flow velocities and MI on INI. Similar to CTR, INI increases with increase in flow velocities. This is because faster replenishment of microbubbles compensates for the decay of signals from microbubbles due to disruption. Thus, signals from microbubbles can last longer until the end of data acquisition. However, INI decreases with increasing MI. This explains the qualitative results in Fig. $3 \mathrm{c}$ and $3 \mathrm{~d}$. While a higher MI gives much higher contrast signals at the beginning of the acquisition, it also tends to destroy the bubbles faster, causing

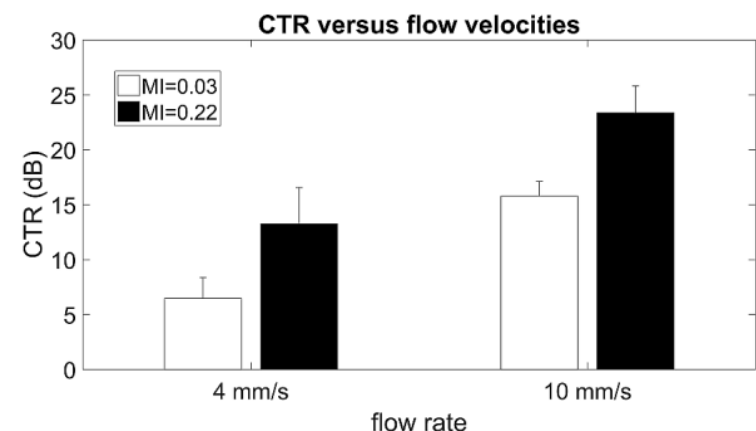

Fig.4. Quantitative evaluation of effects of flow velocity and ultrasound pressure by CTR. CTR increases with increase of flow velocity and MI. Mean value and standard deviation were calculated on three measurements.

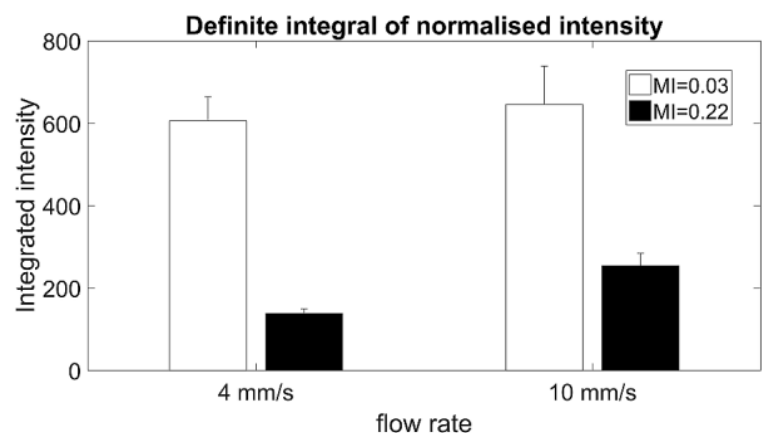

Fig.5. Quantitative evaluation of effects of flow velocity and ultrasound pressure by INI. INI increases with increase of flow velocity and decreases with increase of MI. Mean value and standard deviation were calculated on three measurements.

contrast signals to decrease sharply and they cannot be retained over a long time as shown in Fig. 3d. This is consistent with a smaller INI value obtained which accurately captures such behaviour. INI can correctly capture how long the contrast signals are retained because INI is intensity normalised. Hence, unlike mean CTR, it is not affected by the high contrast at the beginning of the acquisition.

Based on these qualitative and quantitative evaluation, it has been found that the effect of ultrasound pressure depends on the flow velocity. For low flow velocity, differentiation between microbubbles and tissue is difficult due to the similar spatiotemporal coherences. Under this condition, microbubble disruption introduced by increasing ultrasound pressure can significantly contribute to improving contrast. But a compromise between image contrast and persistence should be achieved by optimising the MI value. In the case of low MI, relatively stable microbubbles give insignificant enhancement in contrast quality. While in the case of high MI, bubble signal changes provide much higher contrast signals at the beginning of the acquisition but are decreasing over time due to the diminishing bubble disruption events as bubbles are destroyed with high ultrasound amplitude and replenishment of microbubbles is inadequate. For high flow velocity, contrast quality is already improved by the enhanced difference in spatial and temporal characteristics between fast flowing microbubbles and static tissue.

\section{B. Optical results}

Varied microbubble disruption events under HFR ultrasound with different amplitudes are observed by optical microscope 


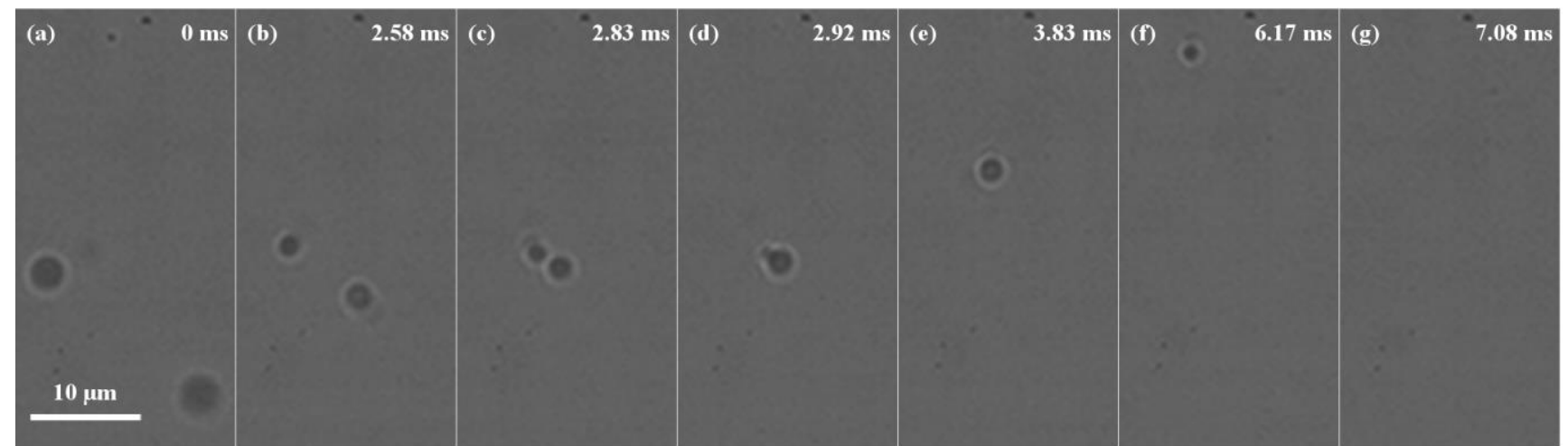

Fig.6. Varied microbubble disruption events under HFR ultrasound (MI=0.36) observed by optical microscope and high speed camera. (a) to (d): Translation and coalescence due to the secondary radiation force; (e) to (f): Translation and diffusion due to the primary radiation force. Scale bar: $10 \mu \mathrm{m}$.

and high speed camera. At lower ultrasound pressure such as $\mathrm{MI}=0.04$ and $\mathrm{MI}=0.02$, microbubbles remain stable and no obvious behaviour are captured. At moderate MI of 0.08 , the main observed behaviour of microbubbles is coalescence. At the higher MI of 0.36 , different disruption events are observed and shown in Fig. 6. Two microbubbles first move to each other and coalesce (Fig. 6a-d) due to the secondary radiation force. At the same time, translation in the direction of ultrasound field and diffusion of coalesced microbubble due to the primary radiation force are observed (Fig. 6e-g).

\section{CONCLUSION}

This work applies HFR CEUS imaging to a lymph mimicking vessel and investigates the effects of flow velocity and ultrasound pressure on HFR CEUS for imaging flow in lymphatic vessel. Our results show that the optimal choice of MI value is dependent on the flow velocity. In particular, MI value has less effect on image quality under high flow velocity while it plays a more significant role at low flow velocity. Under slow flow condition, a high MI value results in high image contrast but low persistence as the bubbles are likely disrupted with slow replenishment. On the other hand, a low MI value disrupts fewer bubbles, allowing for longer persistence at the expense of image contrast. Therefore, an optimal MI value can be chosen to reach a compromise between image contrast and persistence under slow flow condition.

\section{REFERENCES}

[1] "Lymph Node Removal \& Lymphedema," National Breast Cancer Foundation, Inc., 2016. [Online]. Available: http://www.nationalbreastcancer.org/breast-cancer-lymph-noderemoval.

[2] B. B. Goldberg, D. A. Merton, J.-B. Liu, M. Thakur, G. F. Murphy, L. Needleman, A. Tornes and F. Forsberg, "Sentinel Lymph Nodes in a Swine Model with Melanoma:Contrast-enhanced Lymphatic US," Radiology, vol. 230, no. 3, pp. 727-734, 2004.

[3] A. R. Sever, P. Mills, S. E. Jones, K. Cox, J. Weeks, D. Fish and P. A. Jones, "Preoperative Sentinel Node Identification With Ultrasound Using Microbubbles in Patients With Breast Cancer," American Journal of Roentgenology, vol. 196, no. 2, pp. 251-256, 2011.
[4] K. Cox, A. Sever, S. Jones, J. Weeks, P. Mills, H. Devalia, D. Fish and P. Jones, "Validation of a technique using microbubbles and contrast enhanced ultrasound (CEUS) to biopsy sentinel lymph nodes (SLN) in pre-operative breast cancer patients with a normal grey-scale axillary ultrasound," European Journal of Surgical Oncology, vol. 39, no. 7, pp. 760-765, 2013.

[5] M.-X. Tang, N. Kamiyama and R. J. Eckersley, "Effects of nonlinear propagation ultrasound contrast agent imaging," Ultrasound on Med. \& Biol., vol. 36, no. 3, pp. 459-466, 2010.

[6] G. Montaldo, M. Tanter, J. Bercoff, N. Benech and M. Fink, "Coherent Plane-Wave Compounding for very high frame rate ultrasonography and transient elastography," IEEE Transactions on Ultrasonics, Ferroelectrics, and Frequency Control, vol. 56, no. 3, pp. 489-506, 2009.

[7] B. Denarie, T. A. Tangen, I. K. Ekroll, N. Rolim, H. Torp, T. Bjastad and L. Lovstakken, "Coherent Plane Wave Compounding for very high frame rate ultrasonography of rapidly moving targets," IEEE Transactions on Medical Imaging, vol. 32, no. 7, pp. 1265-1276, 2013.

[8] A. C. Yu and L. Lovstakken, "Eigen-based clutter filter design for ultrasound color flow imaging: a review," IEEE Transactions on Ultrasonics, Ferroelectrics, and Frequency Control, vol. 57, no. 5, pp. 1096-1111, 2010.

[9] C. Demene, M. Deffieux, B.-F. Osmanski, V. Biran, J.-L. Gennisson, L.A. Sieu, A. Bergel, S. Franqui, J.-M. Correas, I. Cohen, O. Baud and M. Tanter, "Spatiotemporal Clutter Filtering of Ultrafast Ultrasound Data Highly Increases Doppler and fUltrasound Sensitivity," IEEE Transactions on Medical Imaging, vol. 34, no. 11, pp. 2271-2285, 2015.

[10] Y. Desailly, A.-M. Tissier, J.-M. Correas, F. Wintzenrieth, M. Tanter and O. Couture, "Contrast enhanced ultrasound by real-time spatiotemporal filtering of ultrafast images," Physics in Medicine \& Biology, vol. 62, no. 1, pp. 31-42, 2017.

[11] W. Pan, C. M. Le Roux, S. M. Levy and C. A. Briggs, "The Morphology of the Human Lymphatic Vessels in the Head and Neck," Clinical Anatomy, vol. 23, no. 6, pp. 654-661, 2010.

[12] G. C. Bentel, L. Marks, P. H. Hardenbergh and L. R. Prosnitz, "Variability of the depth of supraclavicular and axillary lymph nodes in patients with breast cancer: is a posterior axillary boost field necessary?," International Journal of Radiation Oncology, Biology, Physics, vol. 47, no. 3, pp. 755-758, 2000.

[13] S. Lin, G. Zhang, C. H. Leow and M.-X. Tang, "Effects of micrchannel confinement on acoustic vaporisation of ultrasound phase change contrast agents," Physics in Medicine \& Biology, vol. 62, no. 17, pp. 6884-6898, 2017.

[14] Robert A. Freitas Jr., "Chapter8: Navigation," in Nanomedicine, Volume I: Basic Capabilities, LANDES BIOSCIENCE, 1999, p. 215. 